\title{
Intramolecular Anti-Hydrosilyation and Silicon-Assisted Cross-Coupling: Highly Regio- and Stereoselective Synthesis of Trisubstituted Homoallylic Alcohols
}

\author{
Scott E. Denmark* and Weitao Pan \\ Roger Adams Laboratory, Department of Chemistry, University of Illinois, \\ Urbana, Illinois 61801
}

\section{SUPPORTING INFORMATION}

All reactions were performed in oven $\left(140^{\circ} \mathrm{C}\right)$ or flame-dried glassware under an inert atmosphere of dry $\mathrm{N}_{2}$. Solvents, pentane and $\mathrm{Et}_{2} \mathrm{O}$, for extraction and chromatography were technical grade and redistilled. Reaction solvents were distilled from the indicated drying agents: methylene chloride $\left(\mathrm{P}_{2} \mathrm{O}_{5}\right)$; diethylether (sodium, benzophenone); hexane (sodium); tetrahydrofuran (THF) (sodium, benzophenone); and toluene (sodium). Flash column chromatography was performed by the method of Still ${ }^{1}$, using 230-400 mesh silica gel purchased from EM Science. $n$-Butyllithium was titrated according to the method of Gilman. ${ }^{2}$ Kugelrohr distillations were performed on a Büchi GKR-50 Kugelrohr; boiling points (bp) correspond to uncorrected air-bath temperatures (ABT); Melting points (mp) were performed in sealed tubes and were determined on a Thomas-Hoover capillary melting point apparatus and were uncorrected.

${ }^{1} \mathrm{H}$ NMR spectra and ${ }^{13} \mathrm{C}$ NMR spectra were recorded on a Varian Unity $500(500 \mathrm{MHz}$, ${ }^{1} \mathrm{H} ; 125.6 \mathrm{MHz},{ }^{13} \mathrm{C}$ ) in $\mathrm{CDCl}_{3}$ (unless otherwise noted). Spectra are referenced to residual chloroform (d $7.26 \mathrm{ppm},{ }^{1} \mathrm{H}$; d $\left.77.23 \mathrm{ppm},{ }^{13} \mathrm{C}\right)$ unless otherwise specified.

Chemical shifts are reported in ppm ( $\delta)$; multiplicities are indicated by s (singlet), d (doublet), t (triplet), q (quartet), qn (quintet), sext (sextet), m (multiplet) and br (broad). Coupling constants, $J$, are reported in Hertz. Mass spectroscopy was performed by the University of Illinois Mass Spectrometer Center. Electron impact (EI) or chemical impact (CI) spectra were performed on a Finnigan-MAT CH-5 spectrometer. Data are reported in the form of m/z (intensity relative to base peak = 100). Infrared spectra (IR) were recorded on a Mattson Galaxy 5020 spectrophotometer. Peaks are reported in $\mathrm{cm}^{-1}$ with indicated relative intensities: $\mathrm{s}$ (strong, 67- 
100\%); m (medium, 34-66\%); w (weak, 0-33\%). Elemental analyses were performed by the University of Illinois Microanalytical Service Laboratory.

Analytical thin-layer chromatography was performed on Merck silica gel plates with QF254 indicator. Visualization was accomplished with UV light and/or iodine. Analytical capillary gas chromatography (GC) was performed on a Hewlett Packard 5890 Series II gas chromatography fitted with a flame ionization detector $\left(\mathrm{H}_{2}\right.$ carrier gas, $\left.1 \mathrm{~mL} / \mathrm{min}\right)$. The columns used are specified in each experiment. The detector temperature was $300{ }^{\circ} \mathrm{C}$. Retention times $\left(t_{R}\right)$ and integrated ratios were obtained from Hewlett Packard 3393A integrators.

Most commercial reagents were purified by distillation or recrystallization prior to use. The 1.0 M solution of TBAF in dioxane was prepared as follows: crystalline TBAF• $3 \mathrm{H}_{2} \mathrm{O}(31.6 \mathrm{~g}$, $100.0 \mathrm{mmol}$ ) was placed into a $100.00 \mathrm{~mL}$ of dry volumetric flask in dry box and the flask was capped with a rubber septum before it was removed from the dry box; dry dioxane was added through syringe to the volume mark.

Silanes $1 \mathbf{a}$ and $\mathbf{1 b}$ were prepared following the literature procedure from the corresponding alkynols. ${ }^{3}\left[\mathrm{RuCl}_{2} \text { (benzene) }\right]_{2}$ was prepared following the literature procedure. ${ }^{4}$ 1-Bromo-4-tertbutyl-1-cyclohexane was prepared following the literature procedure. 5

\section{Preparation of (Z)-3-Ethyliden-2,2-diisopropyl-1-oxa-2-silacyclopentane (3a)}

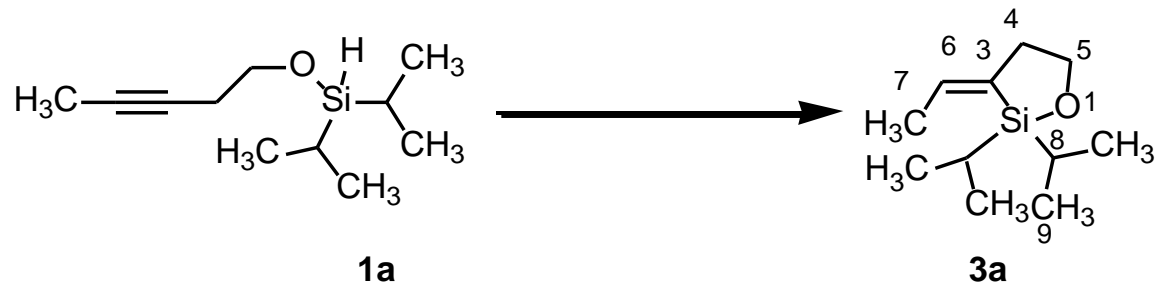

A solution of $\left[\mathrm{RuCl}_{2}\left(\mathrm{C}_{6} \mathrm{H}_{6}\right)\right]_{2}(63 \mathrm{mg}, 0.12 \mathrm{mmol}, 0.063$ equiv) in dry dichloromethane (19 $\mathrm{mL}$ ) was placed into a flask equipped with a condenser, which was affixed with an Y-adapter fitted with a rubber septum and a gas inlet tube. The catalyst was heated to reflux for $10 \mathrm{~min}$. To the refluxing solution was added a solution of silane 1a $(396 \mathrm{mg}, 2.0 \mathrm{mmol})$ in $1.0 \mathrm{~mL}$ of dry dichloromethane by syringe pump over $60 \mathrm{~min}$. The reaction mixture gradually turned red. The solvent was then evaporated in vacuo and pentane $(20 \mathrm{~mL})$ was then added to precipitate the $\mathrm{Ru}$ catalyst. The supernatant was carefully decanted and the residue was extracted with pentane $(2 \times 10$ $\mathrm{mL}$ ) and $10 \mathrm{~mL}$ of pentane/ether, 9/1. The combined organic extracts were filtered through $\mathrm{SiO}_{2}$ $(0.3 \mathrm{~g})$. Removal of solvent afforded $0.33 \mathrm{~g}$ of a pale yellow oil. Kugelrohr distillation of this oil afforded $290 \mathrm{mg}(71 \%)$ of $\mathbf{3 a}$ as a colorless oil. 
Data for 3a:

bp: $\quad 50^{\circ} \mathrm{C}(0.3 \mathrm{mmHg})$

1슬 $: \quad\left(500 \mathrm{MHz}, \mathrm{CDCl}_{3}\right)$

6.31 (qt, $J=2.4,6.6,1 \mathrm{H}, \mathrm{HC}(6)) ; 3.94\left(\mathrm{t}, J=6.6, \mathrm{H}_{2} \mathrm{C}(5), 2 \mathrm{H}\right) ; 2.52-2.49$ (m, $2 \mathrm{H}$, $\mathrm{H}_{2} \mathrm{C}(4)$ ); 1.77 (dt, $J=2.0,6.6,3 \mathrm{H}, \mathrm{H}_{3} \mathrm{C}(7)$ ); 1.16-1.13 (m, $2 \mathrm{H}, \mathrm{HC}(8)$ ); 1.08 (distorted d, $J=6.6,6 \mathrm{H}, \mathrm{H}_{3} \mathrm{C}(9)$ ); 1.03 (distorted d, $J=6.6,6 \mathrm{H}, \mathrm{H}_{3} \mathrm{C}\left(9^{\prime}\right)$ )

${ }^{13}$ C NMR: $\quad\left(125.6 \mathrm{MHz}, \mathrm{CDCl}_{3}\right)$

$136.93(\mathrm{C}(3)), 133.92 \quad(\mathrm{C}(6)), 67.26 \quad(\mathrm{C}(5)), 38.35 \quad(\mathrm{C}(4)), 20.88 \quad(\mathrm{C}(8))$, $\left(17.70 / 17.49\left(\mathrm{C}(9) / \mathrm{C}\left(9^{\prime}\right)\right), 12.96(\mathrm{C}(7))\right.$

IR: (neat)

3014 (m), 3010 (m), 3001 (s), 2943 (s), 2868 (s), 1649 (m), 1464 (m), 1448 (m), $1429(\mathrm{~m}), 1244$ (w), 1059 (m), 1028 (s), 995 (s), 881 (s)

MS: $\quad(\mathrm{EI}, 70 \mathrm{eV})$

$198\left(\mathrm{M}^{+}, 10\right), 173$ (15), 155 (100), 127 (60), 105 (26), 77 (45)

TLC: $\quad R_{f} 0.63$ (pentane/ether, $4 / 1, \mathrm{SiO}_{2}$ )

GC: $\quad t_{R}$ 3a, $7.75 \mathrm{~min}(99.1 \%)$. (Ultra-2, injector $225^{\circ} \mathrm{C}$, column $150{ }^{\circ} \mathrm{C}, 15 \mathrm{psi}$ ).

HRMS: $\quad$ calcd for $\mathrm{C}_{11} \mathrm{H}_{22} \mathrm{OSi}$ : 198.1440 ; found: 198.1443

\section{Preparation of (Z)-3-Pentyliden-2,2-dimethyl-1-oxa-2-silacyclopentane (3b)}

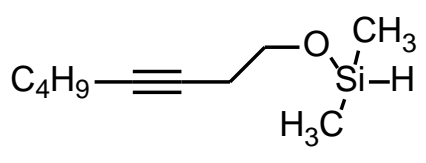

$1 \mathrm{~b}$

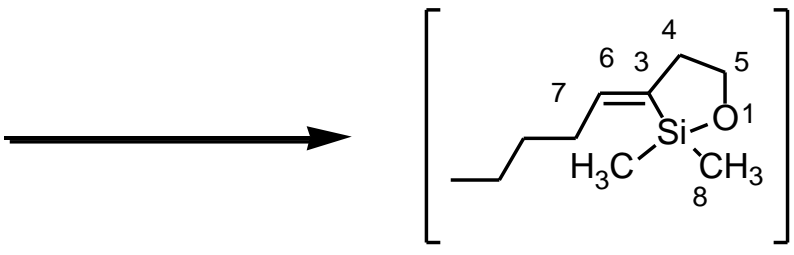

$3 \mathbf{b}$

A solution of $\left[\mathrm{RuCl}_{2}\left(\mathrm{C}_{6} \mathrm{H}_{6}\right)\right]_{2}(400 \mathrm{mg}, 0.80 \mathrm{mmol}, 0.080$ equiv) in dry dichloromethane $(100 \mathrm{~mL})$ was placed into a flask equipped with a condenser, which was affixed with an Y-adapter fitted with a rubber septum and a gas inlet tube. The catalyst was heated to reflux for $10 \mathrm{~min}$. To the reflux solution was added silane $\mathbf{1 b}$ (from $1.26 \mathrm{~g}$ of alcohol $10.0 \mathrm{mmol}$ ) in $4.0 \mathrm{~mL}$ of dry dichloromethane by syringe pump over $150 \mathrm{~min}$. The reaction mixture gradually turned red. The solvent was then evaporated in vacuo and hexane $(100 \mathrm{~mL})$ was then added to precipitate the ruthenium catalyst, which was removed by filtration. The filtrate was then passed through charcoal to remove final traces of ruthenium. After removal of solvent, an yellow oil was obtained (1.63 g, $8.86 \mathrm{mmol}, 86 \%$ ) which was used directly without further purification. 


\section{General Procedure for Coupling of Oxasilacyclopentanes}<smiles>CCCC=C1CCC[As]1(C)C</smiles>

$3 b$

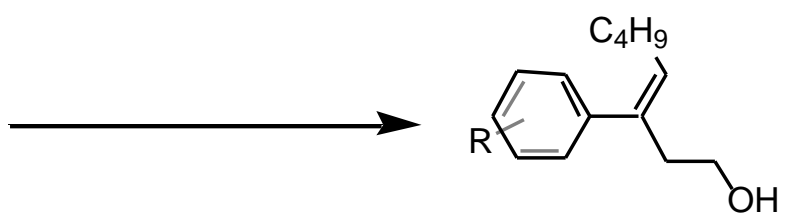

6

In a two-necked flask fitted with a rubber septum and gas inlet tube, was placed a solution of oxasilacyclopentane, $\mathbf{3 b}$ and tetrabutylammonium fluoride (1.0 $\mathrm{M}$ in dioxane), at ambient temperature under nitrogen. The electrophile (1.0 equiv) was added portionwise or in one portion as specified below. The palladium catalyst ( $5 \mathrm{~mol} \%$ of $\mathrm{Pd}$ ) was added in one portion to the mixture following the first portion of electrophile and the mixture was stirred at designated temperature for a designated period of time. The reaction mixture was purified with a specified amount of silica gel as indicated below by column chromatography. The products were further purified by Kugelrohr distillation and/or sublimation.

\section{Preparation of (Z)-3-Phenyl-3-octen-1-ol (6a)}

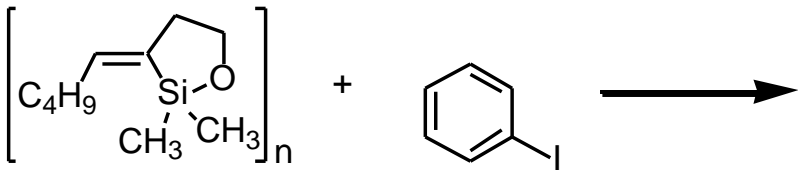

$3 b$

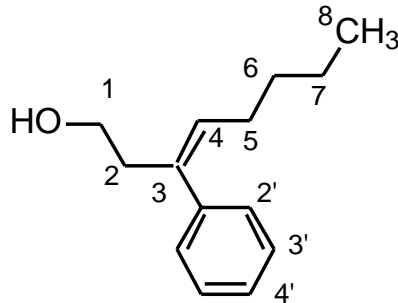

$6 a$

Following the General Procedure, 3b (250 mg, $1.35 \mathrm{mmol}, 1.35$ equiv), was dissolved in a solution of TBAF in dioxane (1.0 M, $2.0 \mathrm{~mL}, 2.0 \mathrm{mmol}, 2.0$ equiv) and the mixture was stirred at $\mathrm{rt}$ for $10 \mathrm{~min}$. Iodobenzene $(204 \mathrm{mg}, 1.0 \mathrm{mmol})$ was added in one portion and $\mathrm{Pd}(\mathrm{dba})_{2}(29.0 \mathrm{mg}$, $0.050 \mathrm{mmol}, 0.050$ equiv) was then added. The mixture was stirred at $45{ }^{\circ} \mathrm{C}$ for $490 \mathrm{~min}$. The solvent was removed in vacuo and the residue was then loaded onto $2 \mathrm{~g}$ of silica gel and was purified by column chromatography $\left(10 \mathrm{~g} \mathrm{SiO}_{2}\right.$, pentane/ether, $\left.1 / 0 \rightarrow 20 / 1 \rightarrow 10 / 1\right)$. Removal of the solvent and Kugelrohr distillation of the resulting yellow oil afforded $133 \mathrm{mg}(65 \%)$ of $\mathbf{6 a}$ as a colorless oil.

Data for 6a:

bp: $\quad 115^{\circ} \mathrm{C}(\mathrm{ABT} 0.1 \mathrm{mmHg})$ 


\section{1ㅅN}

7.38-7.34 (m, $2 \mathrm{H})$; 7.30-7.26 (m, $\left.1 \mathrm{H}, \mathrm{HC}\left(4^{\prime}\right)\right)$; 7.18-7.16 (m, $\left.2 \mathrm{H}\right) ; 5.58(\mathrm{t}, J=7.3$, $1 \mathrm{H}, \mathrm{HC}(4)$ ) ); 3.58 (q, $J=6.1,2 \mathrm{H}, \mathrm{H}_{2} \mathrm{C}(1)$ ); 2.63 (dt, $J=0.8,6.1,2 \mathrm{H}, \mathrm{H}_{2} \mathrm{C}(2)$ ); 1.99 (q, $\left.J=7.1,2 \mathrm{H}, \mathrm{H}_{2} \mathrm{C}(5)\right), 1.38(\mathrm{t}, J=6.1,1 \mathrm{H}, \mathrm{OH}) ; 1.36-1.24(\mathrm{~m}, 4 \mathrm{H}$, $\left.\mathrm{H}_{2} \mathrm{C}(6), \mathrm{H}_{2} \mathrm{C}(7)\right) ; 0.84\left(\mathrm{t}, J=7.3, \mathrm{H}_{3} \mathrm{C}(8), 3 \mathrm{H}\right)$; minor isomer: $5.40(\mathrm{t}, 4.7 \%)$

\section{${ }^{13}$ C NMR: $\quad\left(125.6 \mathrm{MHz}, \mathrm{CDCl}_{3}\right)$}

$140.57,137.20,131.08,128.68,128.44,126.97,60.78$ (C(1)), 42.68, 32.44, 28.91, $22.53,14.19(\mathrm{C}(8))$

IR: $\quad\left(\mathrm{CHCl}_{3}\right)$

3622 (w), 3012 (m), 2970 (s), 2929 (s), 2873 (m), 2858 (m), 1610 (w), 1493 (m), 1466 (m), 1390 (w), 1379 (w), 1230 (w), 1041 (s), 908 (s)

MS: $\quad(\mathrm{EI}, 70 \mathrm{eV})$

$$
204\left(\mathrm{M}^{+}, 43\right), 159 \text { (45), } 143 \text { (100), } 128 \text { (41), } 117 \text { (62), } 105 \text { (17), } 91 \text { (46), } 77 \text { (13) }
$$

TLC: $\quad R_{f} 0.60\left(\mathrm{Et}_{2} \mathrm{O}, \mathrm{SiO}_{2}\right)$

GC: $\quad t_{R}$ 6a, $6.59 \mathrm{~min}$ (Ultra-2, injector $225^{\circ} \mathrm{C}$, detector $300^{\circ} \mathrm{C}$, column $\left.180{ }^{\circ} \mathrm{C}, 15 \mathrm{psi}\right)$

HRMS: $\quad$ calcd for $\mathrm{C}_{14} \mathrm{H}_{20} \mathrm{O}: 204.1514$; found: 204.1515

\section{Preparation of (Z)-3-(2-Methoxyphenyl)-3-octen-1-ol (6b)}

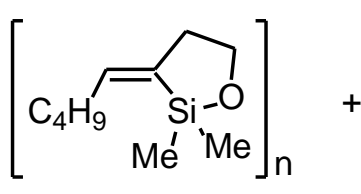

$3 b$
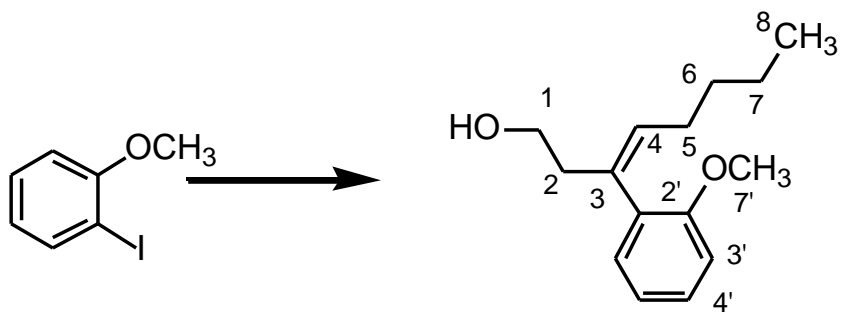

6b

Following the General Procedure, $3 \mathbf{b}$ (235 mg, $1.28 \mathrm{mmol}, 1.28$ equiv) was dissolved in a solution of TBAF in dioxane (1.0 M, $1.0 \mathrm{~mL}, 1.0 \mathrm{mmol}, 1.0$ equiv) and the mixture was stirred at $\mathrm{rt}$ for $10 \mathrm{~min}$. 2-Iodoanisole $\left(235 \mathrm{mg}, 1.0 \mathrm{mmol}\right.$ ) was added followed by $\mathrm{Pd}(\mathrm{dba})_{2}(26 \mathrm{mg}, 0.045$ mmol, 0.045 equiv). The mixture was stirred at $\mathrm{rt}$ for $90 \mathrm{~min}$ before another portion of TBAF in dioxane (1.0 M, $0.50 \mathrm{~mL}, 0.50 \mathrm{mmol}, 0.50$ equiv) was added and the mixture was stirred at $\mathrm{rt}$ for another $27 \mathrm{~h}$ then it was stirred at $35^{\circ} \mathrm{C}$ for $21 \mathrm{~h}$. After evaporation of solvent, the residue was then loaded onto $4 \mathrm{~g}$ of silica gel and was purified by column chromatography ( $30 \mathrm{~g} \mathrm{SiO}_{2}$, pentane/ether, $1 / 0 \rightarrow 20 / 1 \rightarrow 10 / 1)$. Removal of the solvent and Kugelrohr distillation of the resulting oil afforded $160 \mathrm{mg}(68 \%)$ of $\mathbf{6 b}$ as a colorless oil. 
Data for $6 \mathbf{b}$ :

bp: $\quad 135^{\circ} \mathrm{C}(\mathrm{ABT}, 0.15 \mathrm{mmHg})$

1ㅁN

$7.28\left(\mathrm{ddd}, J=1.9,7.3,8.1,1 \mathrm{H}, \mathrm{HC}\left(4^{\prime} / 5^{\prime}\right)\right)$; $7.03\left(\mathrm{dd}, J=1.9,7.3,1 \mathrm{H}, \mathrm{HC}\left(3^{\prime} / 6^{\prime}\right)\right)$; $6.98\left(\mathrm{dt}, J=1.0,8.1,1 \mathrm{H}, \mathrm{HC}\left(5^{\prime} / 4^{\prime}\right)\right), 6.92\left(\mathrm{br} \mathrm{d}, J=8.1,1 \mathrm{H}, \mathrm{HC}\left(6^{\prime} / 3^{\prime}\right)\right) ; 5.66(\mathrm{t}, J=$ 7.3, $1 \mathrm{H}, \mathrm{HC}(4)) ; 3.82$ (s, $\left.3 \mathrm{H}, \mathrm{H}_{3} \mathrm{C}\left(7^{\prime}\right)\right), 3.48$ (t, $\left.J=5.8,2 \mathrm{H}, \mathrm{H}_{2} \mathrm{C}(1)\right)$; 2.56 (br s, 2 $\left.\mathrm{H}, \mathrm{H}_{2} \mathrm{C}(2)\right) ; 1.80$ (q, $J=7.3,2 \mathrm{H}, \mathrm{H}_{2} \mathrm{C}(5)$ ); 1.33-1.21 (m, $4 \mathrm{H}, \mathrm{H}_{2} \mathrm{C}(6), \mathrm{H}_{2} \mathrm{C}(7)$ ); 0.82(t, $\left.J=7.3,3 \mathrm{H}, \mathrm{H}_{3} \mathrm{C}(8)\right)$; minor isomer: 5.55 (t, 4.5\%)

${ }^{13} \underline{\mathrm{C} \mathrm{NMR}}: \quad\left(125.6 \mathrm{MHz}, \mathrm{CDCl}_{3}\right)$

156.63, 133.25, 132.91, 130.34, 129.39, 128.42, 121.11, 110.67, 60.04 (C(1)), 55.61 $\left(\mathrm{C}\left(7^{\prime}\right)\right), 42.68(\mathrm{C}(2)), 31.87,29.09,22.48,14.17(\mathrm{C}(8))$

IR: $\quad\left(\mathrm{CHCl}_{3}\right)$

3545 (w), 3608 (s), 2960 (s), 2935 (s), 2873 (m), 2838 (m), 1599 (m), 1578 (w), 1491 (s), 1435 (s), 1388 (w), 1292 (m), 1279 (m), 1242 (s), 1180 (m), 1115 (m), $1045(\mathrm{~s})$

MS: $\quad(\mathrm{EI}, 70 \mathrm{eV})$

234 ( $\left.\mathrm{M}^{+}, 60\right), 216$ (7), 201 (8), 189 (100), 173 (52), 161 (32), 147 (40), 131 (23), 121 (36), 105 (13), 91 (32), 77 (17)

TLC: $\quad R_{f} 0.44$ (pentane/ether, $2 / 3, \mathrm{SiO}_{2}$ )

GC: $t_{R}$ 6b, $4.76 \min (97 \%)$, minor isomer, $5.15 \min (3 \%)\left(\mathrm{HP}-5\right.$, injector $225{ }^{\circ} \mathrm{C}$, detector $300{ }^{\circ} \mathrm{C}$, column $250{ }^{\circ} \mathrm{C}, 15 \mathrm{psi}$ )

Analysis: $\quad \mathrm{C}_{15} \mathrm{H}_{22} \mathrm{O}_{2}(234.34)$

Calcd.: $\quad$ C, $76.88 ; \quad$ H, $9.46 \%$

Found: $\quad$ C, $76.80 ; \quad H, 9.50 \%$ 


\section{Preparation of Methyl 2-[1-(2-Hydroxyethyl)-(Z)-1-octenyl)]benzoate (6c)}

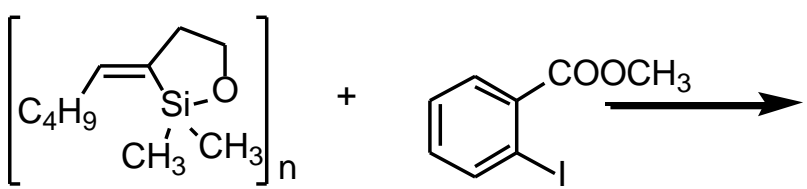

$3 b$

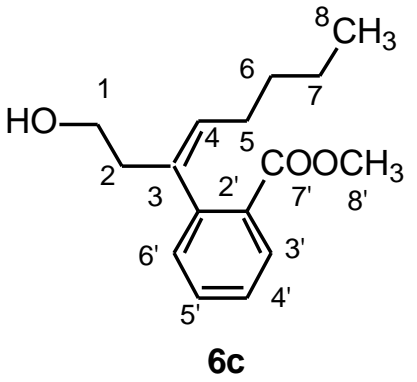

6c

Following the General Procedure, $3 \mathbf{b}$ (238 mg, $1.29 \mathrm{mmol}, 1.28$ equiv), was dissolved in a solution of TBAF in dioxane (1.0 M, $1.0 \mathrm{~mL}, 1.0 \mathrm{mmol}, 1.0$ equiv) and the mixture was stirred at $\mathrm{rt}$ for $10 \mathrm{~min}$. Methyl-2-iodobenzoate was added to the warm solution $\left(35^{\circ} \mathrm{C}\right)$ in three portions over 30 -minute intervals $\left(90.4 \mathrm{mg}, 83.6 \mathrm{mg}, 91.0 \mathrm{mg}\right.$, for a total of $1.01 \mathrm{mmol}, 1.0$ equiv) and $\mathrm{Pd}(\mathrm{dba})_{2}$ (24.6 mg, $0.043 \mathrm{mmol}, 0.042$ equiv) was added following the first portion. The mixture was stirred at $35{ }^{\circ} \mathrm{C}$ for another $43 \mathrm{~h}$. After evaporation of the solvent, the residue was then loaded onto $3 \mathrm{~g}$ of silica gel and was purified by column chromatography (24 $\mathrm{g} \mathrm{SiO}_{2}$, pentane/ether, 19/1 to 5/1). Removal of the solvent and Kugelrohr distillation of the resulting yellow oil afforded $169 \mathrm{mg}$ $(64 \%)$ of $\mathbf{6 c}$ as a pale yellow oil.

Data for 6c:

bp: $\quad 160{ }^{\circ} \mathrm{C}(\mathrm{ABT} 0.15 \mathrm{mmHg})$

1슬 $: \quad\left(500 \mathrm{MHz}, \mathrm{CDCl}_{3}\right)$

$7.85\left(\mathrm{ddd}, J=0.5,1.5,8.2,1 \mathrm{H}, \mathrm{HC}\left(3^{\prime}\right)\right) ; 7.51\left(\mathrm{dt}, J=1.3,7.4,1 \mathrm{H}, \mathrm{HC}\left(5^{\prime}\right)\right) ; 7.34$ $\left(\mathrm{ddd}, J=1.3,7.4,8.0,1 \mathrm{H}, \mathrm{HC}\left(4^{\prime}\right)\right) ; 7.19\left(\mathrm{dt}, J=0.5,8.0,1 \mathrm{H}, \mathrm{HC}\left(6^{\prime}\right)\right) ; 5.56(\mathrm{t}, J=$ 8.0, $1 \mathrm{H}, \mathrm{HC}(4))$; 3.88 (s, $3 \mathrm{H}, \mathrm{H}_{3} \mathrm{C}\left(8^{\prime}\right)$ ); 3.60-3.50 (m, $3 \mathrm{H}, \mathrm{H}_{2} \mathrm{C}(2), \mathrm{H}(-\mathrm{OH})$ ); 2.67-2.64 (m, $2 \mathrm{H}, \mathrm{H}_{2} \mathrm{C}(2)$ ); 1.76-1.71 (m, $\left.2 \mathrm{H}, \mathrm{H}_{2}(5)\right)$; 1.26-1.16 (m, 4 H, $\mathrm{H}_{2} \mathrm{C}(6)$, $\left.\mathrm{H}_{2} \mathrm{C}(7)\right) ; 0.78\left(\mathrm{t}, J=7.3,3 \mathrm{H}, \mathrm{H}_{3} \mathrm{C}(8)\right)$; minor isomer: 5.37 (t, 3.8\%)

${ }^{13} \underline{\mathrm{C} \mathrm{NMR}}: \quad\left(125.6 \mathrm{MHz}, \mathrm{CDCl}_{3}\right)$

168.92 (C(7')), 142.03, 135.69, 132.24, 131.15, 130.87, 130.29, 130.12, 126.98, $59.85(\mathrm{C}(1)), 52.70\left(\mathrm{C}\left(8^{\prime}\right)\right), 43.60,31.62,28.70,22.39,14.05(\mathrm{C}(5))$

IR: $\quad\left(\mathrm{CHCl}_{3}\right)$

3477 (w), 3008 (m), 2956 (S), 2933 (s), 2873 (m), 2862 (m), 1718 (s), 1597 (w), 1570 (w), 1487 (m), 1458 (m), 1435 (m), 1410 (m), 1379 (s), 1294 (s), 1273 (s), 1259 (s), 1219 (m), 1194 (m), 1130 (m), 1093 (s), 1047 (s), 966 (m) 
MS: $\quad(\mathrm{EI}, 70 \mathrm{eV})$

$262\left(\mathrm{M}^{+}, 30\right), 244$ (30), 232 (51), 212 (67), 197 (82), 183 (85), 175 (71), 157 (76), 144 (100), 129 (87), 120 (100), 115 (67), 103 (22), 91 (32), 77 (29)

TLC: $\quad R_{f} 0.53$ (ether, $\mathrm{SiO}_{2}$ )

GC: $\quad t_{R}$ 6c, $7.02 \mathrm{~min}(94.9 \%)$; $t_{R}$ minor isomer, $7.49 \mathrm{~min}(5.1 \%)\left(\mathrm{HP}-5\right.$, injector $225^{\circ} \mathrm{C}$, detector $300{ }^{\circ} \mathrm{C}$, column $270{ }^{\circ} \mathrm{C}, 15 \mathrm{psi}$ )

Analysis: $\quad \mathrm{C}_{16} \mathrm{H}_{22} \mathrm{O}_{2}(262.35)$

Calculated: $\quad$ C, $73.25 ; \quad \mathrm{H}, 8.45$

Found: $\quad$ C, $73.20 ; \quad$ H, 8.79

\section{Preparation of (Z)-3-(2-Nitrophenyl)-3-octen-1-ol (6d)}

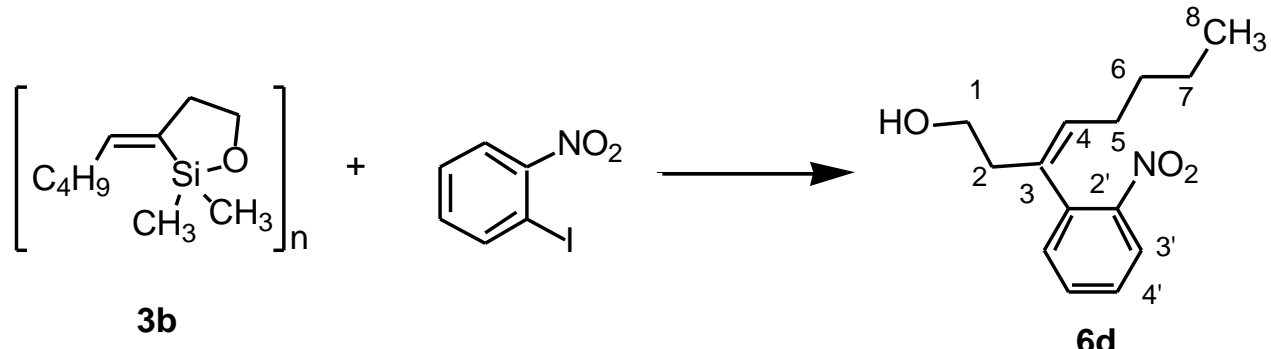

Following the General Procedure, 3b (265 mg, $1.44 \mathrm{mmol}, 1.4$ equiv), was dissolved in a solution of TBAF in dioxane (1.0 M, $1.0 \mathrm{~mL}, 1.0 \mathrm{mmol}, 1.0$ equiv) and the mixture was stirred at $\mathrm{rt}$ for $10 \mathrm{~min}$. 2-Iodonitrobenzene $(249 \mathrm{mg}, 1.0 \mathrm{mmol})$ was added in one portion and $\mathrm{Pd}(\mathrm{dba})_{2}(28.0$ $\mathrm{mg}, 0.053 \mathrm{mmol}, 0.050$ equiv) was then added. The mixture was stirred at $\mathrm{rt}$ for $15 \mathrm{~h}$. Then, an additional $0.25 \mathrm{~mL}$ of TBAF $(0.25 \mathrm{mmol})$ was added and stirring continued for another $7 \mathrm{~h}$. The mixture was then heated at $40{ }^{\circ} \mathrm{C}$ for $24 \mathrm{~h}$ before another portion of TBAF $(0.25 \mathrm{~mL}, 0.25 \mathrm{mmol}$, 0.25 equiv) was added. The heating was continued for anther $23 \mathrm{~h}$. After evaporation of the solvent, the residue was loaded onto $3 \mathrm{~g}$ of silica gel and was purified by column chromatography (32 $\mathrm{g} \mathrm{SiO}_{2}$, pentane/ether, 9/1 to 1/1). Removal of the solvent and Kugelrohr distillation of the resulting yellow oil afforded $166 \mathrm{mg}(66 \%)$ of $\mathbf{6 d}$ as an yellow oil. 
Data for 6d:

bp: $\quad 170{ }^{\circ} \mathrm{C}(\mathrm{ABT} 0.1 \mathrm{mmHg})$

1슬 $: \quad\left(500 \mathrm{MHz}, \mathrm{CDCl}_{3}\right)$

$7.96\left(\mathrm{dd}, J=1.2,8.0,1 \mathrm{H}, \mathrm{HC}\left(3^{\prime}\right)\right) ; 7.62\left(\mathrm{dt}, J=1.5,7.6,1 \mathrm{H}, \mathrm{HC}\left(5^{\prime}\right)\right) ; 7.46$ (ddd, $J$ $\left.=1.5,7.68 .0,1 \mathrm{H}, \mathrm{HC}\left(4^{\prime}\right)\right) ; 7.28\left(\mathrm{dd}, J=1.2,7.6,1 \mathrm{H}, \mathrm{HC}\left(6^{\prime}\right)\right) ; 5.64(\mathrm{t}, J=7.3,1$ $\mathrm{H}, \mathrm{HC}(4)) ; 3.70-3.66(\mathrm{~m}, 2 \mathrm{H}) ; 2.68-2.66(\mathrm{~m}, 2 \mathrm{H}) ; 1.88$ (t, OH, J = 7.3, $1 \mathrm{H},-\mathrm{OH})$; 1.76-1.73 (m, $2 \mathrm{H}), 1.28-1.19(\mathrm{~m}, 4 \mathrm{H}), 0.80\left(\mathrm{t}, J=7.3,3 \mathrm{H}, \mathrm{H}_{3} \mathrm{C}(8)\right)$; minor isomer: $5.50(\mathrm{t}, 3.8 \%)$

${ }^{13} \mathrm{C} \mathrm{NMR}: \quad\left(125.6 \mathrm{MHz}, \mathrm{CDCl}_{3}\right)$

$149.36,135.96$ (2 C), 133.16, 132.38, 131.73, 128.27, 124.65, 60.51 (C(1)), $42.16(\mathrm{C}(2)), 31.66,29.10,22.40,14.50(\mathrm{C}(8))$

IR: $\quad\left(\mathrm{CHCl}_{3}\right)$

3616 (w), 3010 (w), 2960 (m), 2931 (m), 2873 (m), 2862 (m), 1608 (w), 1572 (w), $1527(\mathrm{~s}), 1466(\mathrm{w}), 1354(\mathrm{~s}), 1051(\mathrm{~m})$

MS: $\quad(\mathrm{EI}, 70 \mathrm{eV})$

$249\left(\mathrm{M}^{+}, 1\right), 232$ (5), 214 (10), 202 (12), 176 (36), 160 (30), 146 (32), 130 (37), 120 (100), 104 (37), 91 (30), 77 (35)

TLC: $\quad R_{f} 0.41$ (ether, $\mathrm{SiO}_{2}$ )

HRMS: $\quad$ calcd for $\mathrm{C}_{14} \mathrm{H}_{19} \mathrm{NO}_{3}$ : 249.1365; found: 249.1362

GC: $\quad t_{R} \mathbf{6 d}, 12.36 \mathrm{~min}(100 \%)$ (Ultra-2, injector $225^{\circ} \mathrm{C}$, detector $300{ }^{\circ} \mathrm{C}$, column $220^{\circ} \mathrm{C}$, $15 \mathrm{psi})$

Analysis: $\quad \mathrm{C}_{14} \mathrm{H}_{19} \mathrm{NO}_{3}(249.31)$

Calculated: $\quad$ C, 67.45; $\quad$ H, 7.68; $\quad$ N, 5.62\%

Found: $\quad$ C, 67.12; H, 7.79; N, $5.55 \%$

Preparation of (Z)-3-(3-Hydroxymethylphenyl)-3-octen-1-ol (6e)

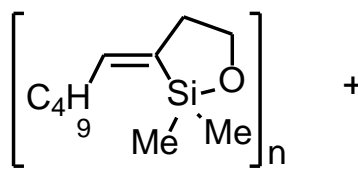

$3 b$

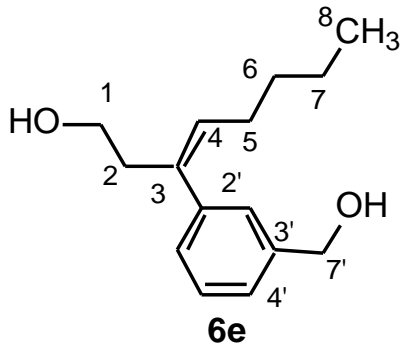

Following the General Procedure, 3b (250 mg, $1.39 \mathrm{mmol}, 1.2$ equiv), was dissolved in a solution of TBAF in dioxane (1.0 M, $2.16 \mathrm{~mL}, 2.16 \mathrm{mmol}, 2.0$ equiv) and the mixture was stirred at 
rt for $10 \mathrm{~min}$. 3-Iodobenzyl alcohol (252 $\mathrm{mg}, 1.08 \mathrm{mmol}$ ) was added followed by $\operatorname{Pd}(\mathrm{dba})_{2}(29.2$ $\mathrm{mg}, 0.051 \mathrm{mmol}, 0.047$ equiv). The mixture was stirred at $\mathrm{rt}$ for a total of $13.5 \mathrm{~h}$ and at $45^{\circ} \mathrm{C}$ for $33 \mathrm{~h}$. After evaporation of the solvent, the residue was loaded onto $2 \mathrm{~g}$ of silica gel and was purified by column chromatography ( $15 \mathrm{~g} \mathrm{SiO}_{2}$, pentane/ether, $\left.1 / 0 \rightarrow 1 / 1 \rightarrow 0 / 1\right)$. Removal of the solvent and Kugelrohr distillation of the resulting oil afforded $148 \mathrm{mg}(59 \%)$ of $\mathbf{6 e}$ as a colorless oil.

Data for 6e:

bp: $\quad 185^{\circ} \mathrm{C}(\mathrm{ABT} 0.1 \mathrm{mmHg})$

${ }^{1}$ H NMR: $\quad\left(500 \mathrm{MHz}, \mathrm{CDCl}_{3}\right)$

7.34 (distorted t, $J=7.6,1 \mathrm{H}, \mathrm{HC}\left(2^{\prime}\right)$ ); 7.27 (distorted d, $J=7.6,1 \mathrm{H}, \mathrm{HC}\left(4^{\prime} / 6^{\prime}\right)$ ); 7.17 (s, $1 \mathrm{H}, \mathrm{HC}\left(2^{\prime}\right)$ ); 7.09 (distorted d, $J=7.6,1 \mathrm{H}, \mathrm{HC}\left(6^{\prime} / 4^{\prime}\right)$ ); 5.57 (t, $J=7.1,1 \mathrm{H}$, $\mathrm{HC}(4)) ; 4.70$ (s, $\left.2 \mathrm{H}, \mathrm{H}_{2} \mathrm{C}\left(7^{\prime}\right)\right) ; 3.55$ (t, $\left.J=6.3,2 \mathrm{H}, \mathrm{H}_{2} \mathrm{C}(1)\right) ; 2.62$ (t, $J=6.3,2 \mathrm{H}$, $\mathrm{H}_{2} \mathrm{C}(2)$ ); 2.12 (br s, OH, $1 \mathrm{H}$ ); 1.97 (q, $J=7.3,2 \mathrm{H}, \mathrm{H}_{2} \mathrm{C}(5)$ ); 1.60 (br s, $1 \mathrm{H}, \mathrm{OH}$ ); 1.36-1.22 (m, $\left.4 \mathrm{H}, \mathrm{H}_{2} \mathrm{C}(6), \mathrm{H}_{2} \mathrm{C}(7)\right)$; $0.83\left(\mathrm{t}, J=7.3,3 \mathrm{H}, \mathrm{H}_{3} \mathrm{C}(8)\right.$ ); minor isomer: $5.82(\mathrm{t}, 1.6 \%)$

${ }^{13}$ C NMR: $\quad\left(125.6 \mathrm{MHz}, \mathrm{CDCl}_{3}\right)$

141.09, 140.80, 137.02, 131.15, 128.65, 128.03, 127.18, 125.61, $65.50\left(\mathrm{C}\left(7^{\prime}\right)\right)$, $60.82(\mathrm{C}(1)), 42.60(\mathrm{C}(2)), 32.41(\mathrm{C}(2)), 28.94,22.51,14.58(\mathrm{C}(8))$

IR: $\quad\left(\mathrm{CHCl}_{3}\right)$

3610 (m), 3012 (s), 2958 (s), 2929 (s), 2875 (m), 2860 (m), 1603 (w), 1466 (m), $1381(\mathrm{~m}), 1234(\mathrm{~m}), 1184(\mathrm{~m}), 1039(\mathrm{~s})$

MS: $\quad(\mathrm{EI}, 70 \mathrm{eV})$ 234 (M+, 24), 216 (73), 185 (27), 173 (83), 159 (60), 143 (100), 129 (70), 117 (87), 105 (22), 91 (57), 77 (23)

TLC: $\quad R_{f} 0.30$ (ether, $\mathrm{SiO}_{2}$ )

GC: $\quad t_{R} 6 \mathbf{6 e}, 12.62 \mathrm{~min}(100 \%)$ (Ultra-2, injector $225^{\circ} \mathrm{C}$, detector $300{ }^{\circ} \mathrm{C}$, column $220^{\circ} \mathrm{C}$, $15 \mathrm{psi})$

HRMS: $\quad$ calcd for $\mathrm{C}_{15} \mathrm{H}_{22} \mathrm{O}_{2}$ : 234.1620 ; found: 234.1628 
Preparation of (Z)-3-(4-Methoxyphenyl)-3-octen-1-ol (6f)

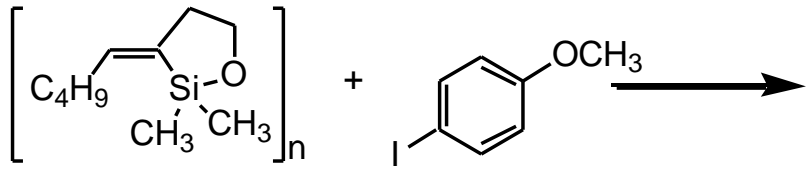

$3 b$

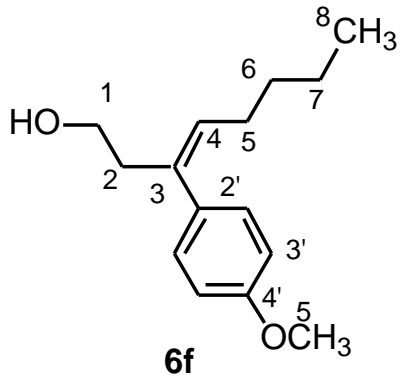

$6 f$

Following the General Procedure, 3b (250 mg, $1.36 \mathrm{mmol}, 1.1$ equiv), was dissolved in a solution of TBAF in dioxane (1.0 M, $1.0 \mathrm{~mL}, 1.0 \mathrm{mmol}, 1.0$ equiv) and the mixture was stirred at $\mathrm{rt}$ for $10 \mathrm{~min}$. 4-Iodoanisole (235 mg, $1.0 \mathrm{mmol}$ ) was added followed by $\mathrm{Pd}(\mathrm{dba})_{2}(27 \mathrm{mg}, 0.047$ mmol, 0.047 equiv). The mixture was stirred at $\mathrm{rt}$ for $30 \mathrm{~min}$ before another portion of TBAF in dioxane was added (1.0 mL, $1.0 \mathrm{mmol}, 1.0$ equiv). The stirring was continued at $\mathrm{rt}$ for $40.5 \mathrm{~h}$ and mixture was then loaded onto $3 \mathrm{~g}$ of silica gel and was purified by column chromatography ( $35 \mathrm{~g}$ $\mathrm{SiO}_{2}$, pentane/ether, $1 / 0 \rightarrow 9 / 1$ ). Removal of the solvent and Kugelrohr distillation of the resulting oil afforded $141 \mathrm{mg}(60 \%)$ of $\mathbf{6 f}$ as a colorless oil.

Data for $6 \mathbf{f}$ :

bp: $\quad 130{ }^{\circ} \mathrm{C}(\mathrm{ABT} 0.10 \mathrm{mmHg})$

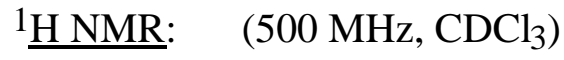

$7.10\left(\mathrm{dt}, J=2.2,8.8,2 \mathrm{H}, \mathrm{HC}\left(2^{\prime}\right)\right) ; 6.90\left(\mathrm{dt}, J=2.2,8.8,2 \mathrm{H}, \mathrm{HC}\left(3^{\prime}\right)\right) ; 5.54(\mathrm{t}, J=$ 6.9, $\mathrm{HC}(4), 1 \mathrm{H}) ; 3.83$ (s, $\left.3 \mathrm{H}, \mathrm{H}_{3} \mathrm{C}\left(5^{\prime}\right)\right) ; 3.56$ (t, $\left.J=6.3,2 \mathrm{H}, \mathrm{H}_{2} \mathrm{C}(1)\right) ; 2.60$ (t, $J=$ 6.3, $2 \mathrm{H}, \mathrm{H}_{2} \mathrm{C}(2)$ ); 1.99 (q, $J=7.3,2 \mathrm{H}, \mathrm{H}_{2} \mathrm{C}(5)$ ); 1.60 (br s, $1 \mathrm{H}, \mathrm{OH}$ ); 1.36-1.25 (m, $\left.4 \mathrm{H}, \mathrm{H}_{2} \mathrm{C}(6), \mathrm{H}_{2} \mathrm{C}(7)\right)$ ) 0.85 (t, $\left.J=7.3,3 \mathrm{H}, \mathrm{H}_{3} \mathrm{C}(8)\right)$; minor isomer: 6.69 (t, $0.7 \%)$

${ }^{13} \mathrm{C}$ NMR: $\quad\left(125.6 \mathrm{MHz}, \mathrm{CDCl}_{3}\right)$

$158.53,136.68,132.76,130.71,129.75,113.81,60.87(\mathrm{C}(1)), 55.43\left(\mathrm{C}\left(5^{\prime}\right)\right), 42.76$ $(\mathrm{C}(2)), 32.51,28.96,22.55,14.52(\mathrm{C}(8))$

IR: $\quad\left(\mathrm{CHCl}_{3}\right)$

3616 (w), 3010 (m), 2960 (s), 2937 (m), 2873 (m), 2858 (m), 2839 (m), 1608 (m), 1512 (s), 1466 (m), 1442 (w), 1379 (w), 1288 (m), 1246 (s), 1176 (m), 1036 (s), $837(\mathrm{~m})$

MS: $\quad(\mathrm{EI}, 70 \mathrm{eV})$

$192\left(\mathrm{M}^{+}, 100\right), 147$ (99), 135 (45), 121 (55), 91 (42), 77 (47)

HRMS: calcd for $\mathrm{C}_{15} \mathrm{H}_{22} \mathrm{O}_{2}$ : 234.1620; found: 234.1620 
TLC: $\quad R_{f} 0.50$ (ether, $2 / 3, \mathrm{SiO}_{2}$ )

GC: $\quad t_{R}$ 6f, $9.92 \mathrm{~min}(100 \%)$ (Ultra-2, injector $225^{\circ} \mathrm{C}$, detector $300{ }^{\circ} \mathrm{C}$, column $270{ }^{\circ} \mathrm{C}$, 15 psi)

Analysis: $\quad \mathrm{C}_{15} \mathrm{H}_{22} \mathrm{O}_{2}(234.34)$

$\begin{array}{lll}\text { Calculated: } & \text { C, } 76.88 ; & \text { H, } 9.46 \% \\ \text { Found: } & \text { C, } 76.50 ; & \text { H, } 9.32 \%\end{array}$

\section{Preparation of 1-[(4-[1-(2-Hydroxyethyl)-(Z)-1-hexenyl)]phenyl]ethanone (6g)}

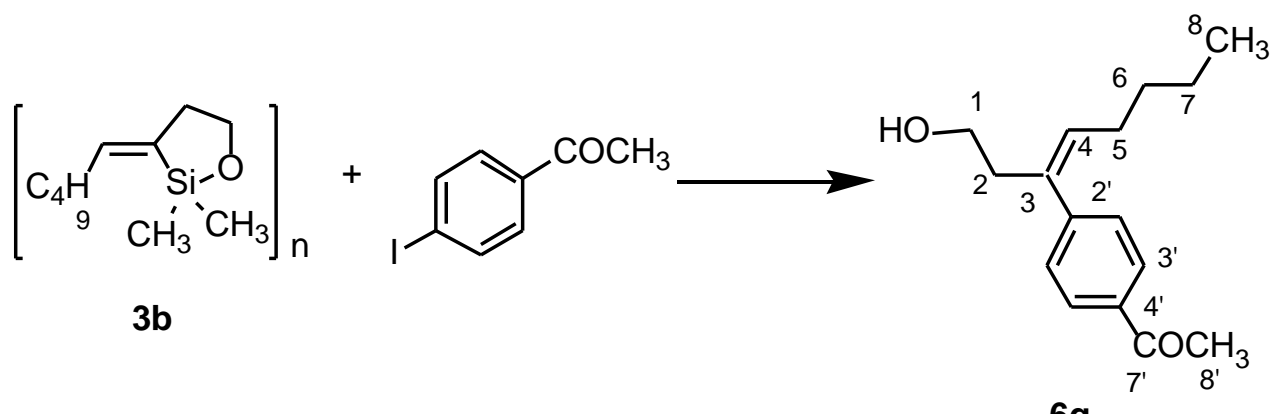

$6 \mathrm{~g}$

Following the General Procedure, 3b (235 mg, $1.28 \mathrm{mmol}, 1.28$ equiv), was dissolved in a solution of TBAF in dioxane (1.0 M, $1.5 \mathrm{~mL}, 1.5 \mathrm{mmol}, 1.5$ equiv) and the mixture was stirred at $\mathrm{rt}$ for $10 \mathrm{~min}$. 4-Iodoacetophenone (249 $\mathrm{mg}, 1.01 \mathrm{mmol}$ ) was added in two portions over 33-min intervals and $\operatorname{Pd}(\mathrm{dba})_{2}(24.5 \mathrm{mg}, 0.043 \mathrm{mmol}, 0.042$ equiv) was added following the first portion of iodide. The mixture was stirred at $\mathrm{rt}$ for a total of $46 \mathrm{~h}$. The crude mixture was then loaded onto 5 $\mathrm{g}$ of silica gel and was purified by column chromatography $\left(40 \mathrm{~g} \mathrm{SiO}_{2}\right.$, pentane/ether, $9 / 1 \rightarrow 7 / 1 \rightarrow$ $4 / 1 \rightarrow 3 / 1$ ). Removal of the solvent and Kugelrohr distillation of the resulting oil afforded $164 \mathrm{mg}$ $(66 \%)$ of $\mathbf{6 g}$ as a colorless oil.

Data for 6g:

bp: $\quad 175^{\circ} \mathrm{C}(\mathrm{ABT} 0.20 \mathrm{mmHg})$

${ }^{1}$ H NMR: $\quad\left(500 \mathrm{MHz}, \mathrm{CDCl}_{3}\right)$

$7.94\left(\mathrm{dt}, J=1.9,8.4,2 \mathrm{H}, \mathrm{HC}\left(3^{\prime}\right)\right) ; 7.26\left(\mathrm{br} \mathrm{d}, J=1.9,8.6,2 \mathrm{H}, \mathrm{HC}\left(2^{\prime}\right)\right)$; 5.62(t, $J=$ 7.5, $1 \mathrm{H}, \mathrm{HC}(4)) ; 3.56$ (t, $J=6.4,2 \mathrm{H}, \mathrm{H}_{2} \mathrm{C}(1)$ ); 2.64-2.61 (m, $2 \mathrm{H}, \mathrm{H}_{2} \mathrm{C}(2)$ ); 2.61 (s, $3 \mathrm{H}, \mathrm{H}_{3} \mathrm{C}\left(6^{\prime}\right)$ ), 1.96 (q, $J=7.5,2 \mathrm{H}, \mathrm{H}_{3} \mathrm{C}(5)$ ); 1.62 (br s, $1 \mathrm{H},-\mathrm{OH}$ ); 1.33-1.32 $(\mathrm{m}, 4 \mathrm{H}, \mathrm{HC}(6), \mathrm{HC}(7)), 0.82\left(\mathrm{t}, J=7.3,3 \mathrm{H}, \mathrm{H}_{3} \mathrm{C}(8)\right)$; minor isomer: $5.95(\mathrm{t}$, $2.8 \%)$ 


\section{${ }^{13}$ C NMR: $\quad\left(125.6 \mathrm{MHz}, \mathrm{CDCl}_{3}\right)$}

$198.15\left(\mathrm{C}\left(5^{\prime}\right)\right), 146.07,136.46,135.80,131.96,128.98,128.60,61.79(\mathrm{C}(1)), 42.24$ $(\mathrm{C}(2)), 32.25,28.93,26.81,22.46,14.10(\mathrm{C}(8))$

IR: $\quad\left(\mathrm{CHCl}_{3}\right)$

3620 (w), 3469 (w), 3010 (s), 2960 (s), 2931 (s), 2873 (m), 2860 (m), 1680 (s), 1558 (w), 1466 (m), 1429 (m), 1379 (m), 1360(s), 1306 (m), 1269 (s), 1182 (m), $1041(\mathrm{~s}), 1016(\mathrm{~m}), 958(\mathrm{~m}), 847(\mathrm{~m}), 837(\mathrm{~m})$

MS: $\quad(\mathrm{EI}, 70 \mathrm{eV})$

$246\left(\mathrm{M}^{+}, 70\right), 231$ (14), 215 (15), 201 (22), 185 (100), 175 (25), 157 (22), 143 (45), 128 (32), 115 (25), 105 (8), 84 (47)

TLC: $\quad R_{f} 0.44$ (ether, $\mathrm{SiO}_{2}$ )

GC: $\quad t_{R}$ 6g, $7.04 \mathrm{~min}(97.4 \%)$; $t_{R}$ minor isomer, $8.73 \mathrm{~min}(2.6 \%)\left(\mathrm{HP}-5\right.$, injector $225{ }^{\circ} \mathrm{C}$, detector $300{ }^{\circ} \mathrm{C}$, column $270{ }^{\circ} \mathrm{C}, 15 \mathrm{psi}$ ).

Analysis: $\quad \mathrm{C}_{16} \mathrm{H}_{22} \mathrm{O}_{2}(246.27)$

Calculated: $\quad$ C, $78.01 ; \quad \mathrm{H}, 9.00 \%$

Found: $\quad$ C, $78.05 ; \quad$ H, $9.08 \%$

\section{Preparation of (Z)-3-(4-Nitrophenyl)-3-octen-1-ol (6h)}

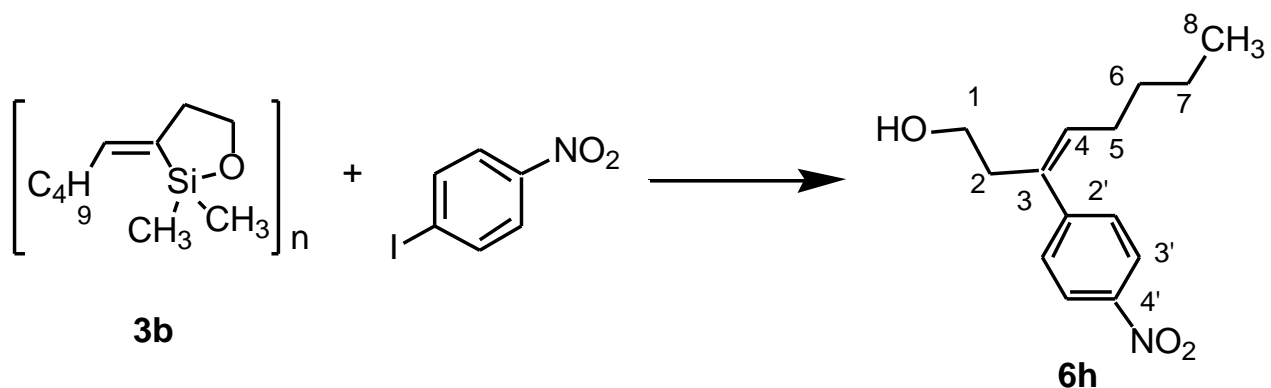

Following the General Procedure, $3 \mathbf{b}$ (250 mg, $1.36 \mathrm{mmol}, 1.1$ equiv), was dissolved in a solution of TBAF in dioxane (1.0 M, $2.0 \mathrm{~mL}, 2.0 \mathrm{mmol}, 2.0$ equiv) and the mixture was stirred at $\mathrm{rt}$ for $10 \mathrm{~min}$. 4-Iodonitrobenzene (252 $\mathrm{mg}, 1.01 \mathrm{mmol}$ ) was added followed by $\mathrm{Pd}(\mathrm{dba})_{2}(29.5 \mathrm{mg}$, $0.051 \mathrm{mmol}, 0.050$ equiv). The mixture was stirred at $45{ }^{\circ} \mathrm{C}$ for a total of $460 \mathrm{~min}$. The crude mixture was then loaded onto $2 \mathrm{~g}$ of silica gel and was purified by column chromatography (10 $\mathrm{g}$ $\mathrm{SiO}_{2}$, pentane/ether, $1 / 0 \rightarrow 9 / 1 \rightarrow 3 / 1$ ). The resulting oil was dissolved in $5 \mathrm{~mL}$ of pentane/ether (9/1) to crystallize out the homo-coupling product. Removal of the solvent and Kugelrohr distillation of the resulting oil afforded $171 \mathrm{mg}(68 \%)$ of $\mathbf{6 h}$ as an yellow oil. 
Data for 6h:

bp: $\quad 195^{\circ} \mathrm{C}(\mathrm{ABT} 0.1 \mathrm{mmHg})$

1슬 $: \quad\left(500 \mathrm{MHz}, \mathrm{CDCl}_{3}\right)$

8.19 (d, $\left.J=8.3,2 \mathrm{H}, \mathrm{HC}\left(3^{\prime}\right)\right) ; 7.32\left(\mathrm{br} \mathrm{d}, J=8.5,2 \mathrm{H}, \mathrm{HC}\left(2^{\prime}\right)\right) ; 5.66(\mathrm{t}, J=7.4,1 \mathrm{H}$, $\mathrm{HC}(4)) ; 3.56$ (t, $\left.J=6.4,2 \mathrm{H}, \mathrm{H}_{2} \mathrm{C}(1)\right) ; 2.62$ (t, $\left.J=6.4,2 \mathrm{H}, \mathrm{H}_{2} \mathrm{C}(2)\right) ; 1.92$ (q, $J=$ 7.4, $2 \mathrm{H}, \mathrm{H}_{2} \mathrm{C}(5)$ ); 1.49 (br s, $\left.J=4.7,1 \mathrm{H}, \mathrm{OH}\right) ; 1.33-1.29$ (m, $2 \mathrm{H}, \mathrm{H}_{2} \mathrm{C}(6)$ ); 1.28 $1.19\left(\mathrm{~m}, 2 \mathrm{H}, \mathrm{H}_{2} \mathrm{C}(7)\right), 0.81\left(\mathrm{t}, J=7.2,2 \mathrm{H}, \mathrm{H}_{3} \mathrm{C}(8)\right)$; minor isomer: $5.98(\mathrm{t}, 4.7 \%)$

${ }^{13} \mathrm{C} \mathrm{NMR}: \quad\left(125.6 \mathrm{MHz}, \mathrm{CDCl}_{3}\right)$

147.98, 146.82, 135.54, 132.74, 129.58, 123.71, 60.99 (C(1)), 41.96, 32.07, 28.82, $22.39,14.88(\mathrm{C}(8))$

IR: $\quad\left(\mathrm{CHCl}_{3}\right)$

3621 (w), 3026 (w), 3012 (w), 2960 (m), 2931 (m), 2875 (w), 2860 (w), 1597 (m), 1520 (s), 1348 (s), 1108 (m), 1043 (w), 1016 (w), 856 (m)

MS: $\quad(\mathrm{EI}, 70 \mathrm{eV})$

249 (M+, 8), 214 (5), 202 (13), 188 (100), 172 (5), 156 (20), 142 (33), 128 (33), 116 (35)

TLC: $\quad R_{f} 0.56$ (ether, $\mathrm{SiO}_{2}$ )

GC: $\quad t_{R} \mathbf{6 h}, 8.83 \min (96.4 \%)$; $t_{R}$ a minor isomer, $10.41 \mathrm{~min}(3.6 \%)$ (HP-5, injector 225 ${ }^{\circ} \mathrm{C}$, detector $300{ }^{\circ} \mathrm{C}$, column $270{ }^{\circ} \mathrm{C}, 15$ psi)

Analysis: $\quad \mathrm{C}_{14} \mathrm{H}_{19} \mathrm{NO}_{3}(249.31)$

Calculated: $\quad$ C, 67.45; $\quad \mathrm{H}, 7.68 ; \quad \mathrm{N}, 5.62 \%$

Found: $\quad$ C, 67.19; H, 7.76; N, 5.74\%

Preparation of 4-[1-(2-Hydroxyethyl)-(Z)-1-hexenyl)]benzonitrile (6i)

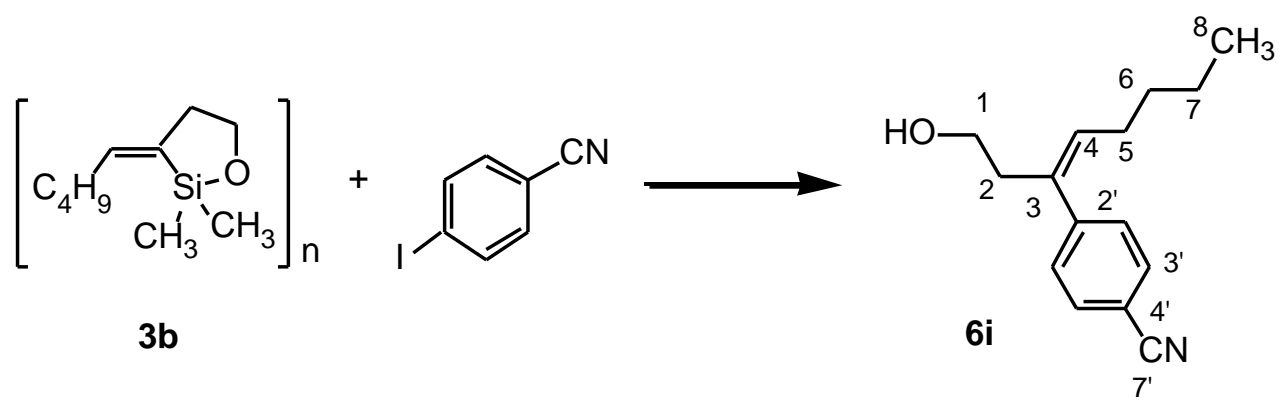

Following the General Procedure, $3 \mathbf{b}$ (250 mg, $1.36 \mathrm{mmol}, 1.36$ equiv), was dissolved in a solution of TBAF in dioxane (1.0 M, $2.0 \mathrm{~mL}, 2.0 \mathrm{mmol}, 2.0$ equiv) and the mixture was stirred at $\mathrm{rt}$ for $10 \mathrm{~min}$. 4-Iodobenzonitrile $(229 \mathrm{mg}, 1.00 \mathrm{mmol})$ was added in one portion and $\mathrm{Pd}(\mathrm{dba})_{2}(26$ 
$\mathrm{mg}, 0.050 \mathrm{mmol}, 0.050$ equiv) was added. The mixture was stirred at $\mathrm{rt}$ for a total of $25.5 \mathrm{~h}$. The crude mixture was then loaded onto $2 \mathrm{~g}$ of silica gel and was purified by column chromatography ( $\mathrm{SiO}_{2}$, pentane/ether, $1 / 0 \rightarrow 10 / 1 \rightarrow 5 / 1 \rightarrow 3 / 1$ ). Removal of the solvent and Kugelrohr distillation of the resulting product afforded $147 \mathrm{mg}(64 \%)$ of $\mathbf{6} \mathbf{i}$ as a colorless oil.

Data for 6i:

bp: $\quad 130{ }^{\circ} \mathrm{C}(\mathrm{ABT} 0.003 \mathrm{mmHg})$

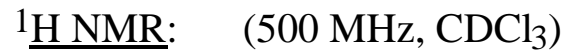

$7.65\left(\mathrm{~d}, J=8.1,2 \mathrm{H}, \mathrm{HC}\left(3^{\prime}\right)\right) ; 7.29$ (d, $\left.J=8.1,2 \mathrm{H}, \mathrm{HC}\left(2^{\prime}\right)\right) ; 5.65(\mathrm{t}, J=7.3,1 \mathrm{H}$, $\mathrm{HC}(4)) ; 3.57$ (t, $\left.J=6.3,2 \mathrm{H}, \mathrm{H}_{2} \mathrm{C}(1)\right) ; 2.62$ (t, $\left.J=6.3,2 \mathrm{H}, \mathrm{H}_{2} \mathrm{C}(2)\right) ; 1.94$ (q, $J=$ 7.3, $2 \mathrm{H}, \mathrm{H}_{2} \mathrm{C}(5)$ ); 1.50 (br s, $1 \mathrm{H}, \mathrm{OH}$ ), 1.30-1.21 (m, $4 \mathrm{H}, \mathrm{H}_{2} \mathrm{C}(6), \mathrm{H}_{2} \mathrm{C}(7)$ ); 0.83 (t, $\left.J=7.3,3 \mathrm{H}, \mathrm{H}_{3} \mathrm{C}(8)\right)$

${ }^{13} \underline{\mathrm{C} \mathrm{NMR}}: \quad\left(125.6 \mathrm{MHz}, \mathrm{CDCl}_{3}\right)$

145.94, 135.90, 132.50, 132.32, 129.55, 119.17, 110.76 (-CN), $60.74(\mathrm{C}(1)), 42.04$ $(\mathrm{C}(2)), 32.18(\mathrm{C}(5)), 28.94,22.46,14.12(\mathrm{C}(8))$

IR: $\quad\left(\mathrm{CHCl}_{3}\right)$

3624 (w), 3030 (w), 3012 (w), 2960 (s), 2930 (s), 2873 (m), 2231 (s), 1606 (m), 1043 (m), 845 (s)

MS: $\quad(\mathrm{EI}, 70 \mathrm{eV})$

$229\left(\mathrm{M}^{+}, 13\right), 204$ (7), 182 (20), 168 (100), 154 (24), 142 (31), 130 (16), 116 (27)

TLC: $\quad R_{f} 0.09$ (ether, $\mathrm{SiO}_{2}$ )

GC: $\quad t_{R} \mathbf{6 i}, 12.71 \mathrm{~min}(100 \%)$ (Ultra-2, injector $225^{\circ} \mathrm{C}$, detector $300{ }^{\circ} \mathrm{C}$, column $220^{\circ} \mathrm{C}$, 15 psi)

HRMS: $\quad$ calcd for $\mathrm{C}_{15} \mathrm{H}_{19} \mathrm{NO}$ (229.32): 229.1467 ; found: 229.1468 


\section{Preparation of Ethyl 4-[1-(2-Hydroxyethyl)-(Z)-1-octenyl)]benzoate (6j)}

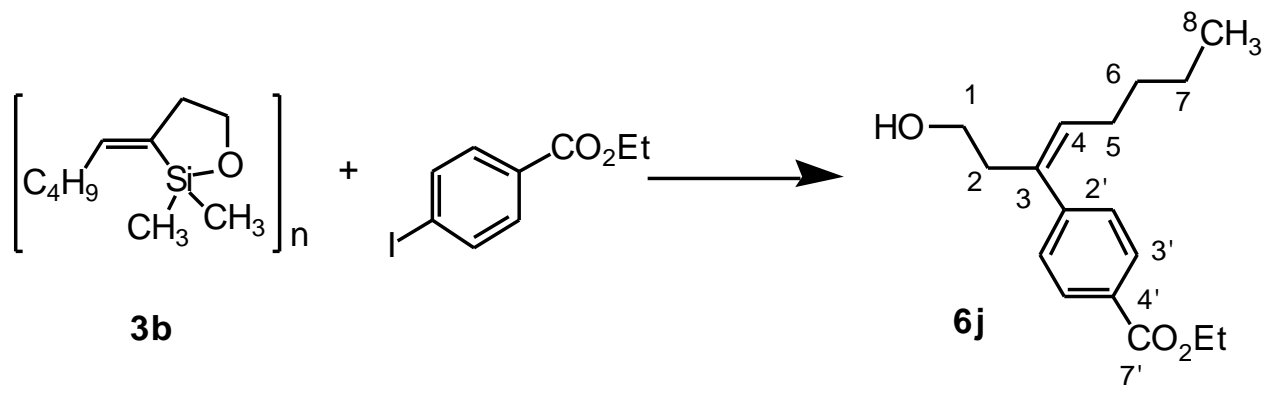

Following the General Procedure, 3b (250 mg, $1.36 \mathrm{mmol}, 1.1$ equiv), was dissolved in a solution of TBAF in dioxane (1.0 M, $1.0 \mathrm{~mL}, 1.0 \mathrm{mmol}, 1.0$ equiv) and the mixture was stirred at $\mathrm{rt}$ for $10 \mathrm{~min}$. Ethyl 4-iodobenzoate $(277 \mathrm{mg}, 1.00 \mathrm{mmol})$ was added followed by $\mathrm{Pd}(\mathrm{dba})_{2}(29 \mathrm{mg}$, $0.05 \mathrm{mmol}, 0.050$ equiv). Another $1.0 \mathrm{~mL}$ of TBAF (1.0 mmol, 1.0 equiv) was added to the mixture after it was stirred at $\mathrm{rt}$ for $10 \mathrm{~min}$. The mixture was then stirred at $40{ }^{\circ} \mathrm{C}$ for a total of 15.5 $\mathrm{h}$, whereupon another $12 \mathrm{mg}$ of $\mathbf{3 b}$ was added. The heating was continued for $4 \mathrm{~h}$, then the crude mixture was loaded onto $2 \mathrm{~g}$ of silica gel and was purified by column chromatography $\left(\mathrm{SiO}_{2}, 10 \mathrm{~g}\right.$, pentane/ether, $1 / 0 \rightarrow 19 / 1 \rightarrow 4 / 1)$. Removal of the solvent and Kugelrohr distillation of the resulting product afforded $200 \mathrm{mg}(72 \%)$ of $\mathbf{6 j}$ as a colorless oil.

Data for 6j:

bp: $\quad 160{ }^{\circ} \mathrm{C}(\mathrm{ABT} 0.1 \mathrm{mmHg})$

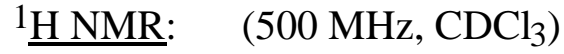

$8.03\left(\mathrm{dd}, J=1.7,6.6, \mathrm{HC}\left(3^{\prime}\right), 2 \mathrm{H}\right) ; 7.24\left(\mathrm{dt}, J=1.7,6.6, \mathrm{HC}\left(2^{\prime}\right), 2 \mathrm{H}\right) ; 5.61(\mathrm{t}, J=$ 7.3, $\mathrm{HC}(4), 1 \mathrm{H}) ; 4.39$ (q, $\left.J=7.0,2 \mathrm{H}, \mathrm{H}_{2} \mathrm{C}\left(6^{\prime}\right)\right) ; 3.56\left(\mathrm{t}, J=6.3,2 \mathrm{H}, \mathrm{H}_{2} \mathrm{C}(1)\right) ; 2.63$ (t, $\left.J=6.3,2 \mathrm{H}, \mathrm{H}_{2} \mathrm{C}(2)\right) ; 1.95$ (q, $\left.J=7.3,2 \mathrm{H}, \mathrm{H}_{2} \mathrm{C}(5)\right) ; 1.62$ (br s, $\left.1 \mathrm{H}, \mathrm{OH}\right), 1.41$ (t, $\left.J=7.0,3 \mathrm{H}, \mathrm{H}_{3} \mathrm{C}\left(7^{\prime}\right)\right)$; 1.39-1.29 (m, $\left.2 \mathrm{H}, \mathrm{H}_{2} \mathrm{C}(6)\right)$; 1.26-1.22 (m, $2 \mathrm{H}, \mathrm{H}_{2} \mathrm{C}(7)$ ); $0.82\left(\mathrm{t}, J=7.3,3 \mathrm{H}, \mathrm{H}_{3} \mathrm{C}(8)\right)$; minor isomer: $5.95(\mathrm{t}, 3.5 \%)$

${ }^{13} \mathrm{C} \mathrm{NMR}: \quad\left(125.6 \mathrm{MHz}, \mathrm{CDCl}_{3}\right)$

$166.68\left(\mathrm{C}\left(5^{\prime}\right)\right), 145.56,136.45,131.70,129.64,128.99,128.63,61.08,60.67,42.21$ (C(2)), 32.18 (C(5)), 28.83, 22.38, 14.49, 14.03

IR: $\quad\left(\mathrm{CHCl}_{3}\right)$

3628 (w), 3012 (w), 2960 (w), 2931 (m), 2875 (w), 2860 (w), 1711 (s), 1608 (w), 1402 (w), 1369 (w), 1308 (m), 1277 (s), 1219 (w), 1178 (m), 1105 (m), 1022 (m), $860(\mathrm{w})$ 
$\underline{\mathrm{MS}}: \quad(\mathrm{EI}, 70 \mathrm{eV})$

$276\left(\mathrm{M}^{+}, 27\right), 231$ (31), 215 (55), 203 (11), 185 (22), 143 (30), 129 (25), 117 (20), 84 (100)

TLC: $\quad R_{f} 0.56$ (ether, $\mathrm{SiO}_{2}$ )

GC: $\quad t_{R} \mathbf{6 j}, 8.92 \mathrm{~min}(96.5 \%) ; t_{R}$ a minor isomer, $10.73 \mathrm{~min}(3.5 \%)$ (HP-5, injector 225 ${ }^{\circ} \mathrm{C}$, detector $300{ }^{\circ} \mathrm{C}$, column $270{ }^{\circ} \mathrm{C}, 15$ psi)

Analysis: $\quad \mathrm{C}_{17} \mathrm{H}_{24} \mathrm{O}_{3}(276.38)$

Calculated: $\quad$ C, $71.78 ; \quad \mathrm{H}, 7.74 \%$

Found: $\quad$ C, $71.85 ; \quad$ H, $7.86 \%$

Preparation of (E)-3-(4-tert-Butyl-1-cyclohexenyl)-3-octen-1-ol (6k)

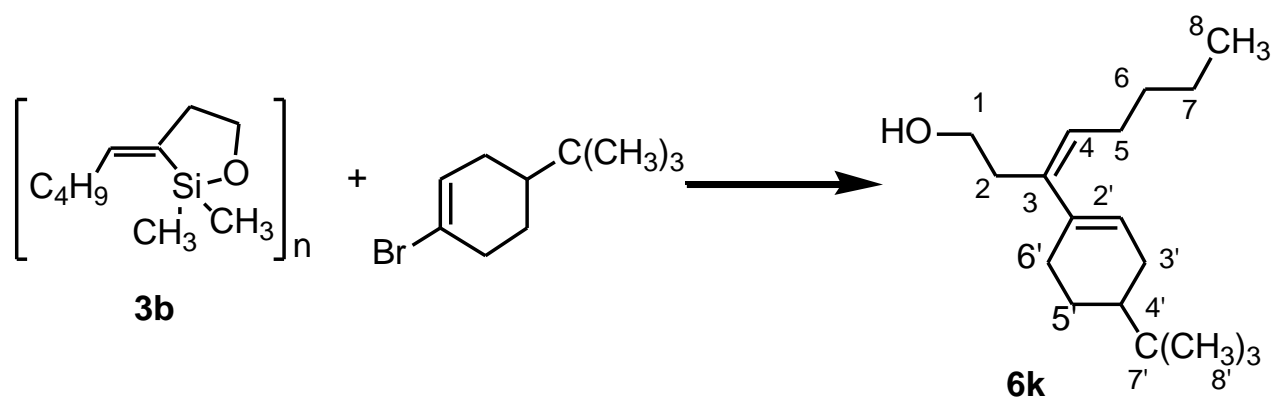

Following the General Procedure, $3 \mathbf{b}$ (230 mg, $1.36 \mathrm{mmol}, 1.36$ equiv), was dissolved in a solution of TBAF in dioxane (1.0 M, $1.0 \mathrm{~mL}, 1.0 \mathrm{mmol}, 1.0$ equiv) and the mixture was stirred at $\mathrm{rt}$ for $10 \mathrm{~min}$. 1-Bromo-4-tert-butyl-1-cyclohexene 5 (226 mg, $1.0 \mathrm{mmol}$ ) was added in one portion and $[\text { allylPdCl}]_{2}(9.0 \mathrm{mg}, 0.025 \mathrm{mmol}, 0.050$ equiv of $\mathrm{Pd})$ was then added. The mixture was stirred at $40{ }^{\circ} \mathrm{C}$ for a total of 3 days. Another portion of [allylPdCl $]_{2}(4.5 \mathrm{mg}, 0.013 \mathrm{mmol}, 0.025$ equiv of $\mathrm{Pd}$ ) was added and stirring at $40{ }^{\circ} \mathrm{C}$ was continued for another $22 \mathrm{~h}$. The crude mixture was then loaded onto $3 \mathrm{~g}$ of silica gel and was purified by column chromatography $\left(10 \mathrm{~g} \mathrm{SiO}_{2}\right.$, pentane/ether, $1 / 0 \rightarrow 20 / 1)$. Removal of the solvent and Kugelrohr distillation of resulting product afforded 85 $\mathrm{mg}(32 \%)$ of $\mathbf{6 k}$ as a colorless oil.

Data for 6k:

mp: $\quad 56-58{ }^{\circ} \mathrm{C}$ 
${ }^{1}$ H NMR: $\quad\left(500 \mathrm{MHz}, \mathrm{CDCl}_{3}\right)$

5.42-5.41 (m, HC(2'), $1 \mathrm{H}) ; 5.24$ (q, $J=7.3,1 \mathrm{H}, \mathrm{HC}(4)) ; 3.60(\mathrm{t}, J=6.1,2 \mathrm{H}$, $\left.\mathrm{H}_{2} \mathrm{C}(1)\right) ; 2.32$ (t, $\left.J=6.2,2 \mathrm{H}, \mathrm{H}_{2} \mathrm{C}(2)\right) ; 2.14-2.02(\mathrm{~m}, 4 \mathrm{H}), 1.90-1.82(\mathrm{~m}, 2 \mathrm{H})$; 1.55 (br s, $1 \mathrm{H}, \mathrm{OH}) ; 1.34-1.14(\mathrm{~m}, 6 \mathrm{H}) ; 1.20$ (m, $2 \mathrm{H}), 0.90-0.88\left(\mathrm{~m}, 9 \mathrm{H}, \mathrm{H}_{3} \mathrm{C}(8)\right.$, $\left.\mathrm{H}_{3} \mathrm{C}\left(8^{\prime}\right)\right)$

${ }^{13}$ C NMR: $\quad\left(125.6 \mathrm{MHz}, \mathrm{CDCl}_{3}\right)$

$139.40\left(\mathrm{C}\left(1^{\prime} / 3\right)\right), 136.49\left(\mathrm{C}\left(3 / 1^{\prime}\right)\right), 129.18,125.51,61.04(\mathrm{C}(1)), 44.19,40.00$, $32.68,32.48,29.98,28.69,27.44,27.06,24.52,22.58,14.28(\mathrm{C}(5))$

IR: $\quad\left(\mathrm{CHCl}_{3}\right)$

3695 (w), 3616 (w), 2974 (s), 2929 (s), 1602 (w), 1457 (m), 1384 (m), 1242 (m), $1111(\mathrm{~s}), 1044(\mathrm{~m})$

MS: $\quad(\mathrm{EI}, 70 \mathrm{eV})$

$264\left(\mathrm{M}^{+}, 72\right), 219$ (27), 207 (60), 193 (15), 189 (22), 177 (24), 163 (32), 149 (17), 133 (23), 119 (39), 105 (41), 91 (59), 79 (37), 67 (34), 57 (100)

TLC: $\quad R_{f} 0.61$ (ether, $\mathrm{SiO}_{2}$ )

GC: $\quad t_{R} \mathbf{6 k}, 11.10 \mathrm{~min}(100 \%)$ (Ultra-2, injector $225^{\circ} \mathrm{C}$, detector $300{ }^{\circ} \mathrm{C}$, column $220^{\circ} \mathrm{C}$, $15 \mathrm{psi})$

HRMS: $\quad$ calcd for $\mathrm{C}_{18} \mathrm{H}_{32} \mathrm{O}: 264.2453$; found: 264.2447

\section{Preparation of (E)-3-(3-Pyridyl)-3-octen-1-ol (6l)}

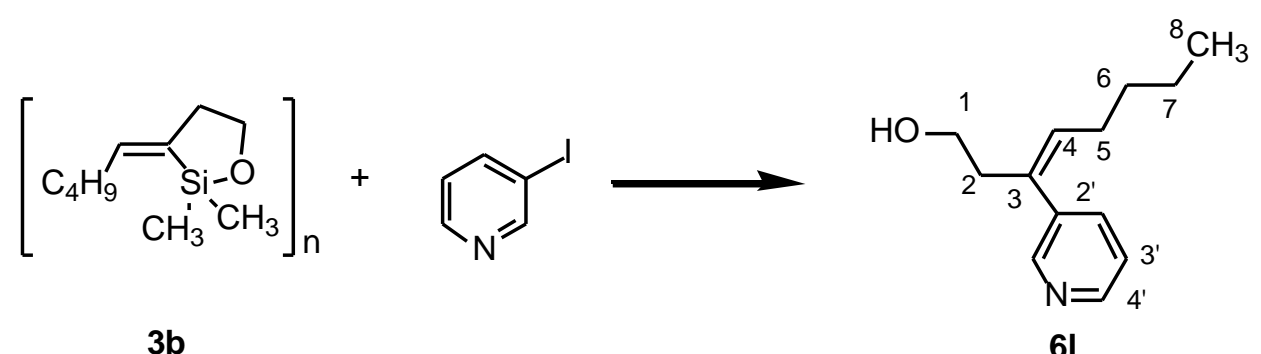

Following the General Procedure, $3 \mathbf{b}$ ( $250 \mathrm{mg}, 1.36 \mathrm{mmol}, 1.36$ equiv), was dissolved in a solution of TBAF in dioxane (1.0 M, $1.5 \mathrm{~mL}, 1.5 \mathrm{mmol}, 1.5$ equiv) and the mixture was stirred at $\mathrm{rt}$ for $10 \mathrm{~min}$. 3-Iodopyridine ( $205 \mathrm{mg}, 1.0 \mathrm{mmol})$ was added in one portion and $\mathrm{Pd}(\mathrm{dba})_{2}(29 \mathrm{mg}$, $0.050 \mathrm{mmol}, 0.050$ equiv) was then added. The mixture was stirred at $45^{\circ} \mathrm{C}$ for a total of 7 days. The crude mixture was then loaded onto $2 \mathrm{~g}$ of silica gel and was purified by column chromatography (10 $\mathrm{g} \mathrm{SiO}_{2}$, pentane/ether, $\left.1 / 0 \rightarrow 9 / 1 \rightarrow 4 / 1 \rightarrow 3 / 1 \rightarrow 1 / 1 \rightarrow 1 / 2 \rightarrow 0 / 1\right)$. Removal 
of the solvent and Kugelrohr distillation of resulting product afforded $120 \mathrm{mg}(59 \%)$ of $\mathbf{6 l}$ as a colorless oil.

Data for 6l:

bp: $\quad 150{ }^{\circ} \mathrm{C}(\mathrm{ABT} 0.1 \mathrm{mmHg})$

1슬 $: \quad\left(400 \mathrm{MHz}, \mathrm{CDCl}_{3}\right)$

$8.53\left(\mathrm{~d}, J=3.9,1 \mathrm{H}, \mathrm{HC}\left(6^{\prime}\right)\right) ; 8.44\left(\mathrm{~s}, 1 \mathrm{H}, \mathrm{HC}\left(4^{\prime}\right)\right) ; 7.51(\mathrm{dt}, J=2.0,7.8,1 \mathrm{H}$, $\left.\mathrm{HC}\left(4^{\prime}\right)\right), 7.24\left(\mathrm{dd}, J=4.9,7.8,1 \mathrm{H}, \mathrm{HC}\left(5^{\prime}\right)\right), 5.69$ (q, $\left.J=7.3, \mathrm{HC}(4), 1 \mathrm{H}\right) ; 3.59$ (t, $J$ $\left.=6.3,2 \mathrm{H}, \mathrm{H}_{2} \mathrm{C}(1)\right) ; 2.63\left(\mathrm{t}, J=6.3,2 \mathrm{H}, \mathrm{H}_{2} \mathrm{C}(2)\right) ; 1.97$ (q, $\left.J=7.3,2 \mathrm{H}, \mathrm{H}_{2} \mathrm{C}(5)\right)$; 1.68 (br s, $1 \mathrm{H}, \mathrm{OH}) ; 1.36-1.22\left(\mathrm{~m}, 4 \mathrm{H}, \mathrm{H}_{2} \mathrm{C}(6), \mathrm{H}_{2} \mathrm{C}(7)\right) ; 0.84$ (t, $J=7.0,3 \mathrm{H}$, $\left.\mathrm{H}_{3} \mathrm{C}(8)\right)$; minor isomer: $5.88(\mathrm{t}, 3.3 \%)$

${ }^{13}$ C NMR: $\quad\left(100.5 \mathrm{MHz}, \mathrm{CDCl}_{3}\right)$

149.68, 148.23, 136.23, 133.74, 132.92, 123.47, $60.66(\mathrm{C}(1)), 42.24(\mathrm{C}(2)), 32.27$ (C(5)), 28.94, 22.48, $14.12(\mathrm{C}(8))$

IR: $\quad\left(\mathrm{CHCl}_{3}\right)$

36221 (w), 3008 (m), 2960 (s), 1568 (w), 1475 (w), 1412 (m), $1236(\mathrm{w}), 1028$ (m), $813(\mathrm{w})$

MS: $\quad(\mathrm{EI}, 70 \mathrm{eV})$ 205 (100), 174 (13), 160 (27), 144 (100), 139 (27), 118 (33), 104 (8), 92 (13)

TLC: $\quad R_{f} 0.14$ (ether, $\mathrm{SiO}_{2}$ )

GC: $\quad t_{R}$ 61, $7.61 \mathrm{~min}(96.2 \%), t_{R}$ minor isomer, $5.56 \mathrm{~min}(3.9 \%)\left(\mathrm{HP}-5\right.$, injector $225{ }^{\circ} \mathrm{C}$, detector $300{ }^{\circ} \mathrm{C}$, column $270{ }^{\circ} \mathrm{C}, 15 \mathrm{psi}$ )

HRMS: $\quad$ calcd for $\mathrm{C}_{10} \mathrm{H}_{13} \mathrm{NO}$ : 205.1467 ; found: 205.1468

\section{References}

(1) Still, W. C.; Kahn, M.; Mitra, A. J. Org. Chem. 1978, 43, 2923

(2) Gilman, H.; Cartledge, F. K.; Sin, S.-Y. J. Organomet. Chem. 1963, 1, 8.

(3) Denmark, S. E.; Pan, W. Org. Lett. 2001, 3, 61.

(4) Bennett, M. A.; Smith, A. K. J. Chem. Soc. Dalton Trans. 1974, 233.

(5) Napolitano, E.; Fiaschi, R.; Mastrorilli, E. Synthesis 1986, 122. 